\title{
Design and Performance Analysis of Mobility Management Schemes Based on Pointer Forwarding for Wireless Mesh Networks
}

\author{
Yinan Li and Ing-Ray Chen, Member, IEEE
}

\begin{abstract}
We propose efficient mobility management schemes based on pointer forwarding for wireless mesh networks (WMNs) with the objective to reduce the overall network traffic incurred by mobility management and packet delivery. The proposed schemes are per-user-based, i.e., the optimal threshold of the forwarding chain length that minimizes the overall network traffic is dynamically determined for each individual mobile user, based on the user's specific mobility and service patterns. We develop analytical models based on stochastic Petri nets to evaluate the performance of the proposed schemes. We demonstrate that there exists an optimal threshold of the forwarding chain length, given a set of parameters characterizing the specific mobility and service patterns of a mobile user. We also demonstrate that our schemes yield significantly better performance than schemes that apply a static threshold to all mobile users. A comparative analysis shows that our pointer forwarding schemes outperform routing-based mobility management protocols for WMNs, especially for mobile Internet applications characterized by large traffic asymmetry for which the downlink packet arrival rate is much higher than the uplink packet arrival rate.
\end{abstract}

Index Terms-Mobility management, pointer forwarding, wireless mesh networks, performance analysis, stochastic Petri net.

\section{INTRODUCTION}

W IRELESS Mesh Networks (WMNs) are gaining rapidly growing interest in recent years, and are widely acknowledged as an innovative solution for next-generation wireless networks. Compared with traditional wireless and mobile networks, e.g., Wi-Fi-based wireless networks and mobile IP networks, WMNs have the advantages of low cost, easy deployment, self-organization and self-healing, and compatibility with existing wired and wireless networks through the gateway/bridge function of mesh routers. A WMN consists of mesh routers and mesh clients [1]. Mesh routers are similar to ordinary routers in wired IP networks, except that they are connected via (possibly multichannel multiradio) wireless links. Mesh clients are wireless mobile devices, e.g., PDAs, smart phones, laptops, etc.

A major expected use of WMNs is as a wireless backbone for providing last-mile broadband Internet access [2] to mesh clients in a multihop way, through the gateway that is connected to the Internet. Because mesh clients may move within a WMN and change their points of attachment frequently, mobility management is a necessity for WMNs to function appropriately. Mobility management consists of location management and handoff management [3]. Location management keeps track of the location information of mesh clients, through location registration and location update operations. Handoff management maintains ongoing connections of mesh clients while they are moving around and changing their points of attachment.

- The authors are with the Department of Computer Science, Virginia Polytechnic Institute and State University, 7054 Haycock Rd, Falls Church,VA 22043. E-mail: yinan926@vt.edu,irchen@cs.vt.edu.

Manuscript received 5 Aug. 2009; revised 13 Jan. 2010; accepted 23 Jan. 2010; published online 27 Aug. 2010.

For information on obtaining reprints of this article, please send e-mail to: tmc@computer.org, and reference IEEECS Log Number TMC-2009-08-0322. Digital Object Identifier no. 10.1109/TMC.2010.166.
Mobility management has been studied intensively for cellular networks and mobile IP networks. A large variety of mobility management schemes and protocols have been proposed for these types of networks over the past years. Comprehensive surveys of mobility management in cellular networks and mobile IP networks can be found in [3] and [4], respectively. Due to some significant differences in network architecture, however, mobility management schemes proposed for cellular networks and mobile IP networks are generally not appropriate for WMNs. For example, the lack of centralized management facilities, e.g., HLR/VLR in cellular networks and HA/FA in mobile IP networks, makes a large portion of the schemes proposed for those types of networks not directly applicable to WMNs, as argued in [1]. Therefore, the development of new mobility management schemes, which take into consideration of the unique characteristics of WMNs, is interesting and important. Additionally, mobility management schemes that are on a per-user basis are highly desired. A per-user-based mobility management scheme can apply specific optimal settings to individual mobile users such that the overall network traffic incurred by mobility management and packet forwarding is minimized. The optimal settings of each mobile user should depend on the user's specific mobility and service patterns, and should be computationally easy to determine.

In this paper, we develop two per-user-based mobility management schemes for WMNs, namely, the static anchor scheme and dynamic anchor scheme. Both schemes are based on pointer forwarding, i.e., a chain of forwarding pointers is used to track the current location of a mesh client. The optimal threshold of the forwarding chain length is determined for each individual mesh client dynamically based on the mesh client's specific mobility and service patterns. 
We develop analytical models based on stochastic Petri nets to evaluate the performance of the proposed schemes. Using the stochastic Petri net models, we demonstrate that for both schemes, there exists an optimal threshold of the forwarding chain length that minimizes the overall network traffic incurred by mobility management and packet forwarding when given a set of parameters characterizing the specific mobility and service patterns of a mesh client. We show that our schemes can yield significantly better performance than schemes that apply a static threshold to all mesh clients, especially when a mesh client's mobility rate is relatively high compared to its service rate. Between the two proposed schemes, we show that the dynamic anchor scheme is better in typical network traffic conditions, whereas the static anchor scheme is better when the service rate of a mesh client is considerably high such that the advantage of the dynamic anchor scheme is offset by the extra cost.

We also carry out a comparative performance analysis to compare our schemes with a representative-routing-based mobility management scheme named as Wireless mesh Mobility Management (WMM) [5]. To study the performance of the WMM scheme, we develop an analytical model that is also based on stochastic Petri nets for the WMM scheme. The comparative performance analysis shows that our schemes outperform the WMM scheme, especially when the network traffic is dominated by mobile Internet applications characterized by large traffic asymmetry, i.e., the traffic load on the downlink is much larger than that on the uplink.

The rest of this paper is organized as follows: Section 2 surveys existing mobility management schemes proposed for WMNs and contrasts our work with existing work. Section 3 describes the system model of our proposed schemes and the assumptions made in the paper. Section 4 and Section 5 introduce the static anchor scheme and dynamic anchor scheme, respectively. Performance modeling and performance analysis are carried out in Sections 6 and 7 , respectively. The paper concludes with Section 8 .

\section{Related Work}

Although mobility management has been studied intensively for other types of networks, e.g., cellular networks and mobile IP networks, it is relatively unexplored for WMNs. As suggested in [6], existing mobility management schemes proposed for WMNs fall into three categories, i.e., tunneling-based, routing-based, and multicasting-based.

\subsection{Tunneling-Based Schemes}

Ant [7] is a mobility management protocol that supports intradomain mobility within a WMN. Although the use of MAC-layer events can help Ant speedup handoff, the signaling cost of location updates in Ant is considerably high, because a location update message has to be sent to a central location server every time a mesh client changes its point of attachment. This is especially a severe problem if the average mobility rate of mesh clients is high.

Huang et al. [8] proposed a mobility management for WMNs called $M^{3}$, which combines per-host routing and tunneling to forward packets to mesh clients. The gateway hosts the location database and user profiles in $M^{3}$. Like our schemes, $M^{3}$ is based on pointer forwarding. However, $M^{3}$ adopts a periodic location update approach, and the location update interval is uniform for all mesh clients. In that sense, $M^{3}$ is not a per-user-based mobility management scheme, and therefore, cannot guarantee optimal performance for every mesh client.

\subsection{Routing-Based Schemes}

iMesh [9] is an infrastructure-mode 802.11-based WMN. iMesh adopts a cross-layer approach for mobility management and develops a routing-based mobility management scheme. A link-layer handoff is triggered when a mesh client moves out of the covering area of its current serving mesh router. After the link-layer handoff is completed, the routing protocol used in iMesh, the Optimized Link State Routing (OLSR) protocol, broadcasts an HNA message announcing the new route of the mesh client. Mobility management in iMesh, therefore, incurs significant overhead due to the broadcasting of the HNA message.

MEsh networks with MObility management (MEMO) [10] is the implementation of an applied WMN with support of mobility management. MEMO uses a modified AODV routing protocol, called as AODV-MEMO, for integrated routing and mobility management. Like the Ant scheme, MEMO also adopts MAC-layer triggered mobility management (MTMM). Although this cross-layer design (Layers 2 and 3) helps reducing the handoff latency, the use of flooding by mesh clients to inform correspondence nodes about location handoffs leads to high signaling cost and bandwidth consumption.

A common problem of iMesh and MEMO is that both of them are based on routing protocols proposed for mobile ad hoc networks that rely on broadcasting for route discovery or location change notification, thus excessive signaling overhead is incurred.

WMM [5] is a novel-routing-based mobility management scheme proposed for WMNs. Location cache is used in combination with routing tables in the WMM scheme for integrated routing and location management. Because location update and location information synchronization can be done while mesh routers route packets, the WMM scheme does not incur significant signaling overhead, as in tunneling-based and multicasting-based schemes. Additionally, as discussed in Section 7.3, the WMM scheme can be virtually viewed as a variant of mobility management schemes based on pointer forwarding, since relevant operations in the WMM scheme resemble forwarding pointer setup and reset operations in pointer forwarding approaches. A detailed description of the WMM scheme can be found in Section 7.3.

\subsection{Multicasting-Based Schemes}

SMesh [11] offers a seamless wireless mesh network system to mesh clients, in the sense that mesh clients view the system as a single access point. Fast handoff in SMesh is achieved by using a group of mesh routers to serve a mesh client and multicast traffic to the mesh client during the handoff. This incurs a high signaling cost, which is especially a severe problem when the average mobility rate of mesh clients is high. Management of multicasting groups is also a major source of signaling overhead in SMesh. 


\section{SYSTEM MODEL}

A WMN consists of two types of nodes: mesh routers (MRs) and mesh clients (MCs). MRs are usually static and form the wireless mesh backbone of WMNs. Some MRs also serve as wireless access points (WAPs) for MCs. One or more MRs are connected to the Internet and responsible for relaying Internet traffic to and from a WMN, and such MRs are commonly referred to as gateways. In this paper, we assume that a single gateway exists in a WMN.

In the proposed mobility management schemes, the central location database resides in the gateway. For each $\mathrm{MC}$ roaming around in a WMN, an entry exists in the location database for storing the location information of the $M C$, i.e., the address of its anchor MR (AMR). The AMR of an $\mathrm{MC}$ is the head of its forwarding chain. With the address of an MC's AMR, the MC can be reached by following the forwarding chain. Data packets sent to an MC will be routed to its current AMR first, which then forwards them to the MC by following the forwarding chain. Packet delivery in the proposed schemes simply rely on the routing protocol used. The concept of pointer forwarding [12] comes from mobility management schemes proposed for cellular networks. The idea behind pointer forwarding is minimizing the overall network signaling cost incurred by mobility management operations by reducing the number of expensive location update events. A location update event means sending to the gateway a location update message informing it to update the location database. With pointer forwarding, a location handoff simply involves setting up a forwarding pointer between two neighboring MRs without having to trigger a location update event.

The forwarding chain length of an MC significantly affects the network traffic cost incurred by the mobility management and packet delivery, with respect to the MC. The longer the forwarding chain, the lower rate the location update event, thus the smaller the signaling overhead. However, a long forwarding chain will increase the packet delivery cost because packets have to travel a long distance to reach the destination. Therefore, there exists a trade-off between the signaling cost incurred by mobility management versus the service cost incurred by packet delivery. Consequently, there exists an optimal threshold of the forwarding chain length for each MC. In the proposed schemes, this optimal threshold denoted by $K$ is determined for each individual MC dynamically, based on the MC's specific mobility and service patterns. We use a parameter named as the service to mobility ratio (SMR) of each MC to depict the MC's mobility and service patterns. For an MC with an average packet arrival rate denoted by $\lambda_{p}$ and mobility rate denoted by $\sigma$, its SMR is formally defined as $S M R=\frac{\lambda_{p}}{\sigma}$.

As discussed in [13], Internet traffic, i.e., the traffic between MRs and the gateway, dominates peer-to-peer traffic in WMNs because WMNs are expected to be a lowcost solution for providing last-mile broadband Internet access. Thus, we assume that for any MC, the Internet session arrival rate is higher than the Intranet session arrival rate, and the average duration of Internet sessions is longer than that of Intranet sessions. We use a parameter $\gamma$ to signify the first assumption, and another parameter $\delta$ to signify the second assumption. More specifically, $\gamma$ denotes the ratio of the Internet session arrival rate to the Intranet

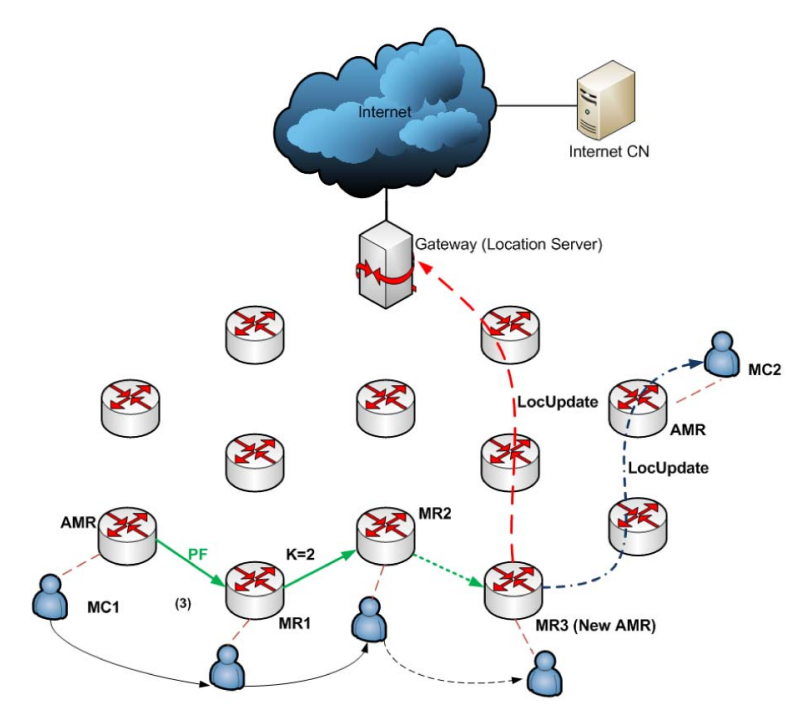

Fig. 1. The handling of location handoffs in the proposed pointer forwarding schemes (LocUpdate means a location update message, and $P F$ means pointer forwarding).

session arrival rate, and $\delta$ denotes the ratio of the average duration of Internet sessions to the average duration of Intranet sessions. In Section 6.3, we show that $\delta$ is also the ratio of the Intranet session departure rate to the Internet session departure rate, using an $M / M / \infty$ queue to model the session arrival process toward an MC.

\section{Static Anchor Scheme}

In the static anchor scheme, an MC's AMR remains unchanged as long as the length of the forwarding chain does not exceed the threshold $K$.

\subsection{Location Handoff}

When an MC moves across the boundary of covering areas of two neighboring MRs, it deassociates from its old serving MR and reassociates with the new MR, thus incurring a location handoff. The MR it is newly associated with becomes its current serving MR. For each MC, if the length of its current forwarding chain is less than its specific threshold $K$, a new forwarding pointer will be set up between the old MR and new MR during a location handoff. On the other hand, if the length of the MC's current forwarding chain has already reached its specific threshold $K$, a location handoff will trigger a location update. During a location update, the gateway is informed to update the location information of the $\mathrm{MC}$ in the location database by a location update message. The location update message is also sent to all the active Intranet correspondence nodes of the MC. After a location update, the forwarding chain is reset and the new MR becomes the AMR of the MC. Fig. 1 illustrates the handling of location handoffs in the proposed schemes.

\subsection{Service Delivery}

\subsubsection{Internet Session}

Internet sessions initiated toward an MC always go through the gateway, i.e., they are always routed to the gateway first before they actually enter into the WMN. Because the 


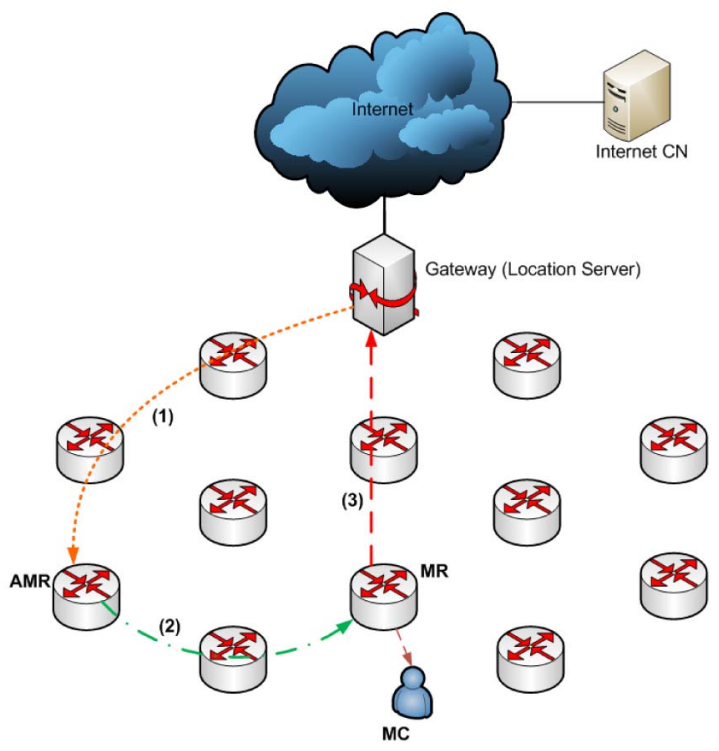

Fig. 2. The location search procedure for newly arrived Internet sessions in the dynamic anchor scheme.

location database resides in the gateway, the gateway always knows the location information of an MC by performing queries in the location database. Therefore, routing an Internet session toward an MC is straightforward. Once the location information of an MC is known, i.e., the address of the MC's AMR is queried, the gateway can route data packets to the AMR, which then forwards them to the $\mathrm{MC}$ by following the forwarding chain.

\subsubsection{Intranet Session}

Unlike Internet sessions, which always go through the gateway where the location database is located, an Intranet session initiated toward an MC within a WMN must first determine the location information of the destination MC through a location search procedure. Suppose a mesh client MC1 initiates an Intranet session toward another mesh client MC2. Upon receiving the new session request from MC1, the serving MR of MC1 (MR1) sends a location query for MC2's location information to the gateway, which performs the query in the location database and replies with the location information of $\mathrm{MC2}$, i.e., the address of the AMR of MC2. After the location search procedure, data packets sent from MC1 to MC2 can be routed directly to the AMR of MC2, which then forwards them to MC2 by following the forwarding chain.

\section{Dynamic Anchor Scheme}

In the dynamic anchor scheme, the current forwarding chain of an MC will be reset due to the arrival of new Internet or Intranet sessions. The idea behind this scheme is to reduce the packet delivery cost by keeping the AMR of an MC close to its current serving MR when the service to mobility ratio is high, thus relieving the problem of triangular routing (gateway-AMR-MC) of the static anchor scheme, with the extra cost of resetting the forwarding chain upon a new session arrival.

The handling of location handoffs in the dynamic anchor scheme is the same as in the static anchor scheme, as shown

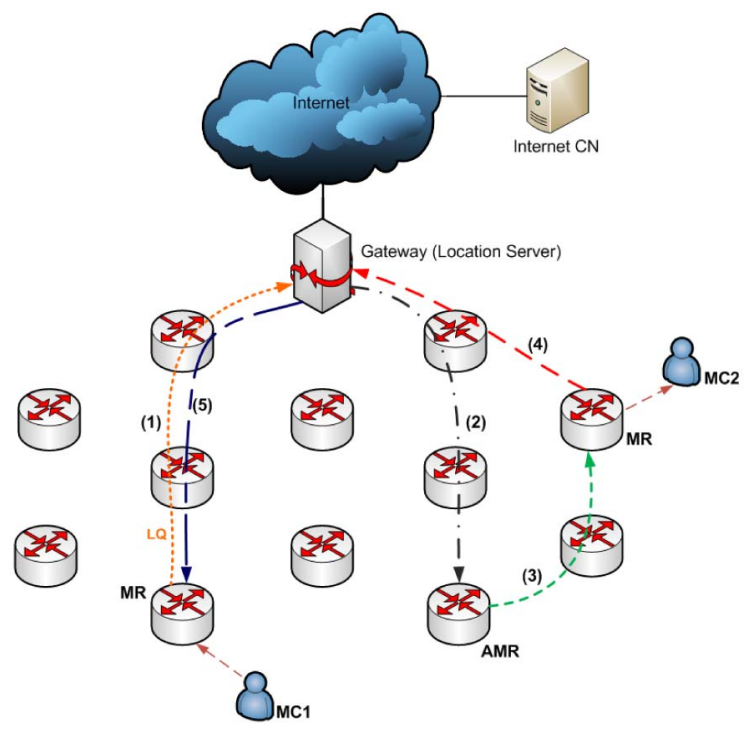

Fig. 3. The location search procedure for newly arrived Intranet sessions in the dynamic anchor scheme.

in Fig. 1. However, the mechanism of service delivery in the dynamic anchor scheme is significantly different from that in the static anchor scheme.

\subsection{Service Delivery}

\subsubsection{Internet Session}

In the dynamic anchor scheme, when a new Internet session toward an MC arrives at the gateway, the gateway will not route the session to the AMR of the MC immediately. Instead, a location search procedure is executed to locate the MC's current serving MR, which may be different from its AMR. Fig. 2 illustrates the location search procedure for newly arrived Internet sessions. Specifically, the gateway sends a location request message to the AMR of the MC, which forwards the location request to its current serving MR. Upon receiving the location request message, the MC's current serving MR sends a location update message to the gateway, announcing that it is the new AMR of the MC. When the gateway receives the location update message, it updates the location information of the MC in the location database, i.e., marking that the current serving $M R$ of the MC becomes its new AMR. After the location search procedure, the forwarding chain is reset and subsequent data packets will be routed to the new AMR of the MC. The gain is that the routing path is shortened, thus reducing the packet delivery cost.

\subsubsection{Intranet Session}

When a new Intranet session is initiated toward an MC, a location search procedure similar to the one above is executed to locate the current serving MR of the destination MC. Fig. 3 illustrates the location search procedure for newly arrived Intranet sessions. Let MC1 and MC2 denote the source mesh client and destination mesh client, respectively. When a new Intranet session initiated toward MC2 by MC1 arrives at the current serving MR of MC1 (MR1), MR1 sends a location request message to the gateway, which queries the location database and routes the location request message to the AMR of MC2, which 
TABLE 1

The Parameters and Notations Used in Performance Modeling and Analysis

\begin{tabular}{|l||l|}
\hline Parameter & Notation \\
\hline$\sigma$ & Mobility rate \\
\hline$\lambda_{I} / \mu_{I}$ & Internet session arrival/departure rate \\
\hline$\lambda_{L} / \mu_{L}$ & Intranet session arrival/departure rate \\
\hline$\lambda_{p u}$ & $\begin{array}{l}\text { Average uplink (outcoming) packet arrival rate of } \\
\text { Internet sessions }\end{array}$ \\
\hline$\lambda_{p d}$ & $\begin{array}{l}\text { Average downlink packet arrival rate of Internet } \\
\text { sessions }\end{array}$ \\
\hline$\lambda_{p L}$ & Average packet arrival rate of Intranet sessions \\
\hline$N_{p I}$ & $\begin{array}{l}\text { Average number of downlink (incoming) packets } \\
\text { per Internet session }\end{array}$ \\
\hline$N_{p L}$ & $\begin{array}{l}\text { Average number of incoming packets per Intranet } \\
\text { session }\end{array}$ \\
\hline$N_{I}$ & $\begin{array}{l}\text { Instantaneous average number of active Internet } \\
\text { correspondence nodes per MC }\end{array}$ \\
\hline$N_{L}$ & $\begin{array}{l}\text { Instantaneous average number of active Intranet } \\
\text { correspondence nodes per MC }\end{array}$ \\
\hline$N$ & $\begin{array}{l}\text { Number of MRs in a WMN } \\
\text { gverage distance (number of hops) between the } \\
\text { gateway and an arbitrary MR }\end{array}$ \\
\hline$\alpha$ & $\begin{array}{l}\text { Average distance (number of hops) between two } \\
\text { arbitrary MRs }\end{array}$ \\
\hline$\beta$ & $\begin{array}{l}\text { Ratio of the Internet session arrival rate to the } \\
\text { Intranet session arrival rate }\end{array}$ \\
\hline$\gamma$ & $\begin{array}{l}\text { Ratio of the average duration of Internet sessions } \\
\text { to the one of Intranet sessions }\end{array}$ \\
\hline$P_{f}$ & $\begin{array}{l}\text { Ratio of the downlink packet arrival rate to the } \\
\text { uplink packet arrival rate of Internet sessions }\end{array}$ \\
\hline$P_{f}$ & $\begin{array}{l}\text { One-hop communication latency between two } \\
\text { neighboring MRs }\end{array}$ \\
\hline$P_{g}$ & $\begin{array}{l}\text { Rate of reconnection when an MC switches from } \\
\text { sleep mode back to active mode }\end{array}$ \\
\hline $\begin{array}{l}\text { Probability that an MC moves forward } \\
\text { gateway due to unknown location information of } \\
\text { the destination MC in the WMM scheme }\end{array}$ \\
\hline $\begin{array}{l}\text { Probability that the location query procedure is } \\
\text { executed in the WMM scheme }\end{array}$ \\
\hline \begin{tabular}{l} 
quest message in the WMM scheme \\
\hline
\end{tabular} & \\
\hline &
\end{tabular}

forwards the location request message to MC2's current serving MR (MR2). Upon receiving the location request message, MR2 replies to the gateway with a location update message, announcing that it is the new AMR of MC2. The location information of MC2 in the location database is updated by the gateway after it receives the location reply. The updated location information of MC2 is sent to MR1 in response to the location request and the location search procedure is completed. After the location search procedure, subsequent data packets will be routed to the new AMR of MC2 directly.

\section{Performance Model}

In this section, we develop analytical models for evaluating the performance of the proposed schemes. The analytical models are built using stochastic Petri nets (SPNs). We choose stochastic Petri nets as the tool for performance modeling because 1) an SPN model is a concise representation of the underlying Markov or semi-Markov chain that may have a large number of states; and 2) an SPN model is capable of reasoning the behavior of an $\mathrm{MC}$, as it migrates

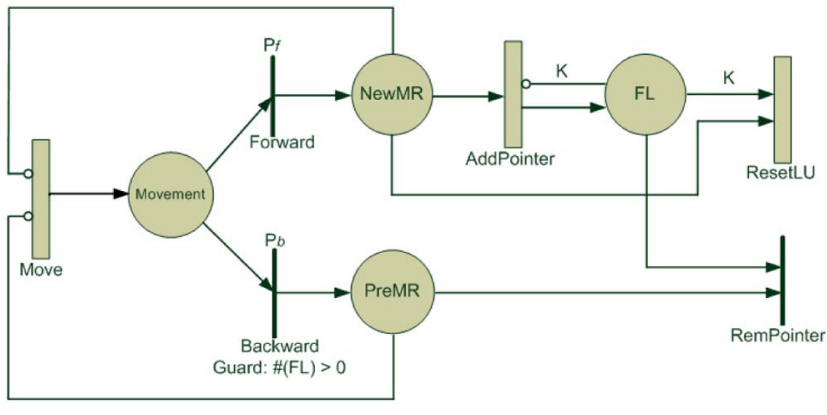

Fig. 4. The SPN model for the static anchor scheme.

TABLE 2

The Interpretation of Places and Transitions Defined in the SPN Model for the Static Anchor Scheme

\begin{tabular}{|l||l|}
\hline Symbol & Interpretation \\
\hline Move & A timed transition modeling MC movement \\
\hline Movement & $\begin{array}{l}\text { Mark (Movement) } 1 \text { means that the MC just } \\
\text { moved }\end{array}$ \\
\hline Forward & $\begin{array}{l}\text { An immediate transition modeling forward } \\
\text { movement }\end{array}$ \\
\hline Backward & $\begin{array}{l}\text { An immediate transition modeling backward } \\
\text { movement }\end{array}$ \\
\hline NewMR & $\begin{array}{l}\text { Mark (newMR) } 1 \text { means that the MC just moved } \\
\text { forward to a new MR }\end{array}$ \\
\hline PreMR & $\begin{array}{l}\text { Mark (PreMR) } 1 \text { means that the MC just moved } \\
\text { backward to the most recently visited MR }\end{array}$ \\
\hline AddPointer & $\begin{array}{l}\text { A timed transition modeling the event of setting } \\
\text { up a forwarding pointer between two neighbor- } \\
\text { ing MRs }\end{array}$ \\
\hline ResetLU & $\begin{array}{l}\text { Mark (F L) indicates the forwarding chain length } \\
\text { A timed transition modeling the event of updat- } \\
\text { ing location database and resetting the for- } \\
\text { warding chain due to MC movement that causes } \\
\text { the threshold } K \text { to be exceeded }\end{array}$ \\
\hline RemPointer & $\begin{array}{l}\text { An immediate transition modeling the event of } \\
\text { removing a forwarding pointer due to a back- } \\
\text { ward movement }\end{array}$ \\
\hline
\end{tabular}

among states in response to system events. Table 1 summarizes the parameters and notations used in the following sections.

\subsection{Static Anchor Scheme}

The SPN model for the static anchor scheme is shown in Fig. 4. This model essentially captures the behaviors of an MC while it is moving around within a WMN. The interpretation of places and transitions defined in the SPN model is given in Table 2. Here, we briefly describe how the SPN model is constructed:

- The movement of an MC is modeled by transition Move, the transition rate of which is represented by the mobility rate $\sigma$ of an MC. When an MC moves to a new MR and is reassociated with it, thus incurring a location handoff, a new token is put into place Movement, meaning that the location handoff is completed.

- An MC can move forward to a new MR or move backward to the most recently visited MR. The SPN model differentiates between these two cases using two immediate transitions, i.e., Forward and Backward. Probabilities $P_{f}$ and $P_{b}$ are associated with Forward 


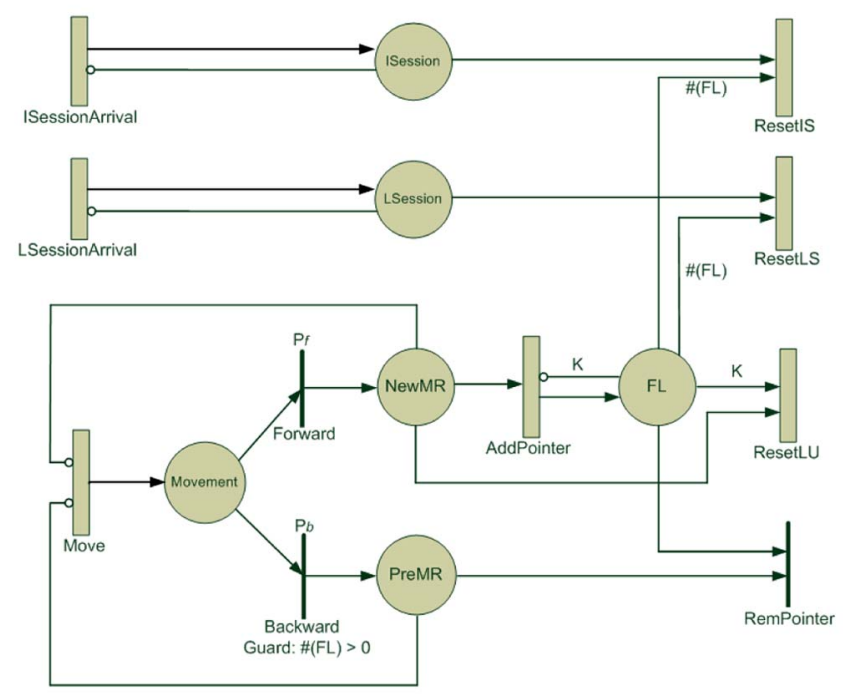

Fig. 5. The SPN model for the dynamic anchor scheme.

and Backward, respectively. The values of $P_{f}$ and $P_{b}$ depend on the network coverage model used, which will be introduced in Section 6.3.

- If an MC moves forward to a new MR, transition Forward is fired and a new token is put into place NewMR. If the current forwarding chain length is smaller than $K$, a new forwarding pointer needs to be set up. This is modeled by enabling and firing transition AddPointer, if the number of tokens in place $F L$ is less than $K$.

- If the number of tokens in place $F L$ is already equal to $K$, a new forward movement triggers a location update and the forwarding chain is reset. This is modeled by firing transition $\operatorname{Reset} L U$, when there are $K$ tokens in place $F L$ and a token in place NewMR. The firing of transition ResetLU will consume all the $K$ tokens in place $F L$, representing that the forwarding chain is reset.

- If an MC moves backward to the most recently visited MR, transition Backward is fired and a token is put in place PreMR. This will subsequently enable and fire immediate transition RemPointer. Removing a pointer upon a backward movement is modeled by an immediate transition as forwarding pointers will be purged automatically.

- Notice that it is only reasonable for an MC to move backward, when the current serving MR of the MC is not its AMR, i.e., the forwarding chain length is not zero. This is modeled by associating an enabling function (Mark (FL) > 0) with transition Backward.

- The inhibitor arcs in the SPN model are used to model the assumption that an MC will not move during a location handoff.

- The transition rates of transition AddPointer and ResetLU are parameterized in Section 6.3.

\subsection{Dynamic Anchor Scheme}

The SPN model for the dynamic anchor scheme is shown in Fig. 5. Because the handling of location handoffs is the same in both schemes, part of this SPN model is identical to the
TABLE 3

The Interpretation of Additional Places and Transitions Defined in the SPN Model for the Dynamic Anchor Scheme

\begin{tabular}{|l||l|}
\hline Symbol & Interpretation \\
\hline ISessionArrival & $\begin{array}{l}\text { A timed transition modeling the arrival of In- } \\
\text { ternet sessions }\end{array}$ \\
\hline LSessionArrival & $\begin{array}{l}\text { A timed transition modeling the arrival of In- } \\
\text { tranet sessions }\end{array}$ \\
\hline ISession & A place holding newly arrived Internet sessions \\
\hline LSession & A place holding newly arrived Intranet sessions \\
\hline ResetIS & $\begin{array}{l}\text { A timed transition modeling resetting the for- } \\
\text { warding chain due to a newly arrived Internet } \\
\text { session }\end{array}$ \\
\hline ResetLS & $\begin{array}{l}\text { A timed transition modeling resetting the for- } \\
\text { warding chain due to a newly arrived Intranet } \\
\text { session }\end{array}$ \\
\hline
\end{tabular}

SPN model for the static anchor scheme. The SPN model for the dynamic anchor scheme has four new transitions and two new places to capture the behavior of the scheme when new sessions arrive toward an MC. Table 3 gives the interpretation of the additional transitions and places. Here, we briefly describe how the additional part of the SPN model for the dynamic anchor scheme is constructed.

- The event of new (Internet or Intranet) session arrival toward an $\mathrm{MC}$ is modeled by firing transition ISessionArrival or LSessionArrival, the transition rates of which are $\lambda_{I}$ and $\lambda_{L}$, respectively. The firing of transition ISessionArrival or LSessionArrival causes a token to be put into place ISession or LSession, depending on the type of the new session.

- In the dynamic anchor scheme, the arrival of a new session causes the current forwarding chain to be reset, and the new MR to become its new AMR. This is modeled by firing transition ResetIS for a newly arrived Internet session or ResetLS for a newly arrived Intranet session. The firing of transition ResetIS or ResetLS consumes all the tokens in place $F L$, modeling that the current forwarding chain is reset upon a new session arrival.

- The inhibitor arcs are used to model the assumption that new sessions will not arrive during the course of a forwarding chain reset.

- The transition rates of transition ResetIS and ResetLS are parameterized in Section 6.3.

\subsection{Parameterization}

Transition AddPointer models the event of setting up a forwarding pointer between two neighboring MRs, which involves a round-trip communication between the two MRs, i.e., the communication cost is $2 \tau$. The transition rate $\mu_{\text {AddPointer }}$ is the reciprocal of the communication delay, i.e.,

$$
\mu_{\text {AddPointer }}=\frac{1}{2 \tau} \text {. }
$$

Transition ResetLU models the event of resetting the forwarding chain of an MC during a location update, which involves updating the MC's location information in the location database, and sending a location update message to each active Intranet correspondence node $(\mathrm{CN})$ of the $\mathrm{MC}$. The signaling cost thus consists of two parts. The first part is 
for the new MR to inform the gateway to update the $\mathrm{MC}^{\prime} \mathrm{s}$ location information in the location database, i.e., $\alpha \tau$. The second part is for the new MR to inform all the active Intranet $\mathrm{CNs}$ of the $\mathrm{MC}$, i.e., $N_{L} \beta \tau$. Thus, the transition rate $\mu_{\text {Reset } L U}$ is

$$
\mu_{\text {Reset } L U}=\frac{1}{\left(\alpha+N_{L} \beta\right) \times \tau} .
$$

Transition ResetIS models the event of resetting the forwarding chain of an MC due to the arrival of a new Internet session. As introduced in Section 5.1, in this event, a location request message is sent from the gateway to the current serving MR of the MC, and a location update message is replied to the gateway in response to the location request. The location update message is also sent to all the active Intranet CNs of the MC. The communication cost in this case is, therefore $\left(2 \alpha+N_{L} \beta+i\right) \times \tau$, where $i$ is the current length of the forwarding chain. Thus, the transition rate $\mu_{\text {ResetIS }}$ is

$$
\mu_{\text {ResetIS }}=\frac{1}{\left(2 \alpha+N_{L} \beta+i\right) \times \tau} .
$$

Transition ResetLS models the event of resetting the forwarding chain of an MC due to the arrival of a new Intranet session. Let $\mathrm{MC} 1$ and $\mathrm{MC} 2$ denote the source $\mathrm{MC}$ and destination $\mathrm{MC}$, respectively. In this event, a location request message is sent from the serving MR (MR1) of MC1 to the current serving MR (MR2) of MC2, forwarded by the gateway. In response to the location request, a location update message is replied by MR2 to the gateway, which then forwards the updated location information of MC2 to MR1. The location update message is also sent to all the active Intranet $\mathrm{CNs}$ of $\mathrm{MC} 2$. The communication cost in this case is thus $\left(4 \alpha+N_{L} \beta+i\right) \times \tau$, where $i$ is the current length of the forwarding chain. Thus, the transition rate $\mu_{\text {Reset } L S}$ is

$$
\mu_{\text {Reset } L S}=\frac{1}{\left(4 \alpha+N_{L} \beta+i\right) \times \tau} .
$$

Immediate transitions Forward and Backward are associated with probabilities $P_{f}$ and $P_{b}$, respectively. These probabilities depend on the network coverage model and the mobility model assumed. In this paper, we assume the square-grid mesh network model for WMNs [14] and the random walk model [15] for MCs. For the square-grid mesh network model, we assume that all MRs have the same wireless range that covers direct neighboring MRs located in four orthogonal directions. Additionally, we consider a relatively large wireless mesh network simulated by a wrapped-around structure such that each MR has four direct neighbors. Under these models, an MC can move randomly from the current MR to one of the MR's four neighbors with equal probability, i.e., $1 / 4$. Thus, $P_{f}$ and $P_{b}$ can be calculated as

$$
P_{f}=\frac{3}{4}, \quad P_{b}=\frac{1}{4} .
$$

Packet arrival rates, e.g., $\lambda_{p u}, \lambda_{p d}$, and $\lambda_{p L}$, are effective rates over the continuous time space. Because packets arrive only when there are on-going sessions, these rates depend on session arrival rates, e.g., $\lambda_{I}$ and $\lambda_{L}$, and average numbers of packets per session, e.g., $N_{p I}$ and $N_{p L}$. We use an $M / M / \infty$ queue to model the process of session arrival toward an MC. The average number of on-going sessions of an $\mathrm{MC}$ at any instance can be obtained using queuing theory, and thus, the effective packet arrival rate can be derived. Specifically, the average number of on-going Internet (Intranet) sessions of an MC denoted by $N_{I}\left(N_{L}\right)$ at any instance is calculated as

$$
\begin{aligned}
& N_{I}=\frac{\lambda_{I}}{\mu_{I}}, \\
& N_{L}=\frac{\lambda_{L}}{\mu_{L}}, \quad \text { with } \quad \lambda_{L}=\frac{\lambda_{I}}{\gamma} \quad \text { and } \quad \mu_{L}=\delta \times \mu_{I} .
\end{aligned}
$$

Notice that in (6), we state that $\mu_{L}=\delta \times \mu_{I}$. This is because according to queuing theory, the ratio of the average duration of Internet sessions to the average duration of Intranet denoted by $\delta$ is defined as $\delta=\frac{1 / \mu_{I}}{1 / \mu_{L}}=\frac{\mu_{L}}{\mu_{I}}$.

The effective downlink (incoming) and uplink (outcoming) packet arrival rates of Internet sessions and the effective packet arrival rate of Intranet sessions are derived as

$$
\begin{aligned}
\lambda_{p d} & =N_{p I} \times N_{I} \times \lambda_{I}, \\
\lambda_{p u} & =\frac{\lambda_{p d}}{\zeta}, \\
\lambda_{p L} & =N_{p L} \times N_{L} \times \lambda_{L} .
\end{aligned}
$$

\subsection{Performance Metrics}

We use the total communication cost incurred per time unit as the metrics for performance evaluation and analysis. The total communication cost includes the signaling cost of location handoff and update operations, the signaling cost of location search operations, and the packet delivery cost. For the static anchor scheme, the signaling cost of location search operations is incurred when a new Intranet session is initiated toward an MC. For the dynamic anchor scheme, the signaling cost of location search operations represents the cost for tracking the current serving MR of an MC and resetting the forwarding chain when new sessions are initiated toward an MC. In the following, we use $C_{\text {static }}$ and $C_{\text {dynamic }}$ to represent the total communication cost incurred per time unit by the static anchor scheme and dynamic anchor scheme, respectively. $C_{\text {location }}, C_{\text {search }}$ and $C_{\text {delivery }}$ are used to represent the signaling cost of a location handoff operation, the signaling cost of a location search operation, and the cost to deliver a packet, respectively. Subscripts are associated with these cost terms. Specifically, subscript "I" and " $L$ " denote Internet and Intranet sessions, respectively. Subscript " $\mathrm{s}$ " and " $\mathrm{d}$ " denote the static anchor scheme and dynamic anchor scheme, respectively.

For the static anchor scheme, the total communication cost incurred per time unit is calculated as

$$
\begin{aligned}
C_{\text {static }}= & C_{\text {location }} \times \sigma+C_{\text {search }, L} \times \lambda_{L} \\
& +C_{\text {delivery }, I} \times \lambda_{p d}+C_{\text {delivery }, L} \times \lambda_{p L} .
\end{aligned}
$$

For the dynamic anchor scheme, the total communication cost incurred per time unit is calculated as

$$
\begin{aligned}
C_{\text {dynamic }}= & C_{\text {location }} \times \sigma+C_{\text {search }, I} \times \lambda_{I} \\
& +C_{\text {search }, L} \times \lambda_{L}+C_{\text {delivery }, I} \times \lambda_{p d} \\
& +C_{\text {delivery }, L} \times \lambda_{p L} .
\end{aligned}
$$


TABLE 4

The Parameters and Their Default Values Used

\begin{tabular}{|c|c||c|c||c|c|}
\hline Parameter & Value & Parameter & Value & Parameter & Value \\
\hline$\gamma$ & 10 & $\delta$ & 5 & $\lambda_{I}$ & $\frac{1}{600}$ \\
\hline$\mu_{I}$ & $\frac{1}{600}$ & $N_{I}$ & 200 & $N_{L}$ & 100 \\
\hline$\alpha$ & 30 & $\beta$ & 30 & $N$ & 1000 \\
\hline$P_{g}$ & $5.0 \%$ & $P_{q}$ & $10.0 \%$ & $P_{r}$ & $50.0 \%$ \\
\hline$\tau$ & 1 & $\omega_{\text {active }}$ & $\frac{1}{1200}$ & $\omega_{\text {sleep }}$ & $\frac{1}{600}$ \\
\hline
\end{tabular}

The stochastic models underlying the SPN models shown in Figs. 4 and 5 are continuous-time Markov chains. Let $P_{i}$ denote the probability that the underlying Markov chain is found in a state that the current forwarding chain length is $i$. Let $S$ denote the set of states in the underlying Markov chain. Then, $C_{\text {location }}$ can be calculated as

$$
C_{\text {location }}=\sum_{S} P_{i} C_{i, \text { location }}
$$

where $C_{i, l o c a t i o n}$ is calculated as

$$
C_{i, \text { location }}= \begin{cases}2 \tau, & \text { if } 1 \leq i<K, \\ \left(\alpha+N_{L} \beta\right) \times \tau, & \text { if } i=K .\end{cases}
$$

The location search cost $C_{\text {search }}$ can be calculated as

$$
C_{\text {search }}=\sum_{S} P_{i} C_{i, \text { search }},
$$

where $C_{i, \text { search }}$ is either $C_{i, \text { search },, L}, C_{i, \text { search }, d, I}$, or $C_{i, \text { search }, d, L}$. The equations for calculating $C_{i, \text { search }, s, L}, C_{i, \text { search }, d, I}$, and $C_{i, \text { search }, d, L}$ are shown as follows:

$$
\begin{aligned}
C_{i, \text { search },, L} & =2 \alpha \tau, \\
C_{i, \text { search }, d, I} & =\left(2 \alpha+N_{L} \beta+i\right) \times \tau, \\
C_{i, \text { search }, d, L} & =\left(4 \alpha+N_{L} \beta+i\right) \times \tau .
\end{aligned}
$$

The packet delivery $\operatorname{cost} C_{\text {delivery }}$ is calculated in a similar way as follows:

$$
C_{\text {delivery }}=\sum_{S} P_{i} C_{i, \text { delivery }},
$$

where $C_{i, \text { delivery }}$ is either $C_{i, \text { delivery,I }}$ or $C_{i, \text { delivery, } L} \cdot C_{i, \text { delivery, } I}$ and $C_{i, d e l i v e r y, L}$ can be calculated as follows:

$$
\begin{aligned}
C_{i, \text { delivery }, I} & =(\alpha+i) \times \tau, \\
C_{i, \text { delivery }, L} & =(\beta+i) \times \tau .
\end{aligned}
$$

These costs can be calculated by associating the above SPN models with reward functions and calculating the steady-state rewards, using the SPNP [16] package.

\section{Performance Analysis}

In this section, we analyze the performance of the proposed schemes, in terms of the total communication cost incurred per time unit. Additionally, we compare the proposed schemes with two baseline schemes. In the first baseline scheme, pointer forwarding is not used, meaning that every movement of an MC will trigger a location update event. Thus, it is essentially the same as having $K=0$ in the proposed schemes. In the second baseline scheme, pointer

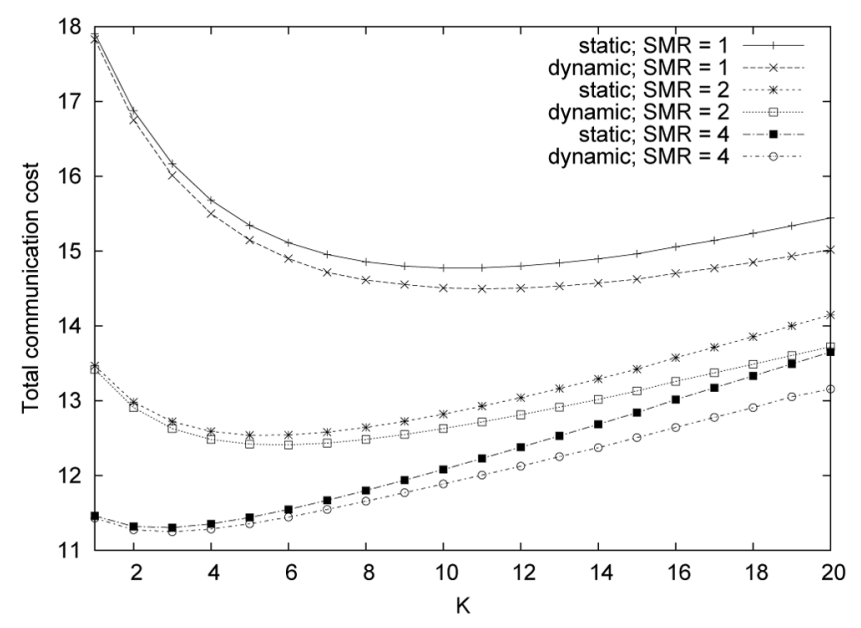

Fig. 6. Total communication cost versus $K$.

forwarding is employed, but the same threshold of the forwarding chain length is preset for all MCs, e.g., $K=4$ for all MCs. We also carry out the performance comparison between our schemes and the WMM scheme proposed in [5]. A detailed description of the WMM scheme and the SPN model constructed for it will be given in Section 7.3. Table 4 lists the parameters and their default values used in the performance evaluation. The time unit used is second. All costs presented below are normalized with respect to $\tau=1$.

\subsection{Proposed Pointer Forwarding Schemes}

Fig. 6 shows the total communication cost as a function of $K$ in both schemes, under different SMRs. As shown in the figure, for both schemes, there exists an optimal threshold $K$ that results in minimized total communication cost. For example, when $S M R=1$, the optimal $K$ is 10 for the static anchor scheme, whereas it is 11 for the dynamic anchor scheme. Another observation is that the total communication cost in both schemes decreases, as SMR increases. This is because given fixed session arrival rates, the mobility rate decreases as SMR increases, thus the signaling cost incurred by location management as well as the total communication cost decreases.

It is interesting to note in Fig. 6 that the dynamic anchor scheme always performs better than the static anchor scheme, under the given parameter values in Table 4 and the investigated SMRs. However, since, the dynamic anchor scheme incurs additional overhead of resetting the forwarding chain of an MC upon session arrival, it is expected that in cases that session arrival rates are considerably high, the additional overhead will offset its advantage. This is demonstrated by Fig. 7, which plots the cost difference between the static anchor scheme and dynamic anchor scheme, as a function of SMR, with $\lambda_{I}=\frac{1}{30}$ and $\mu_{I}=\frac{1}{30}$.

It can be seen in Fig. 7 that, initially, when SMR is small, the dynamic anchor scheme performs better than the static anchor scheme. However, as SMR increases, there exists a crossover point beyond which the static anchor scheme starts performing better than the dynamic anchor scheme. It is interesting to see that there exists another crossover point of SMR beyond which the dynamic anchor scheme is superior again. This is because when SMR is considerably 


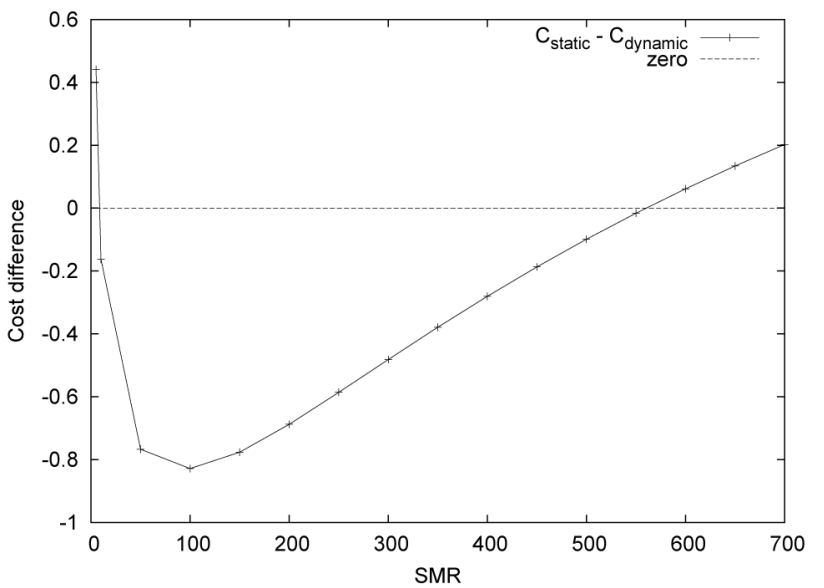

Fig. 7. $C_{\text {static }}-C_{\text {dynamic }}$ versus SMR.

large, i.e., when the mobility rate is considerably small relatively to the session arrival rate, resetting the forwarding chain due to new session arrival in the dynamic anchor scheme essentially makes the AMR of an MC be the same as its current serving MR most of the time, thus significantly reducing the packet delivery cost. It is worth noting that because the total communication cost is a per time unit measure, the accumulative effect of even a small cost difference will be significant.

Fig. 8 plots the optimal threshold $K$ as a function of SMR in both schemes. It can be observed that for both schemes, the optimal $K$ decreases, as SMR increases. This is because as SMR increases, with fixed session arrival rates, the mobility rate decreases; thus a short forwarding chain is favorable to reduce the service delivery cost. It is also interesting to see that the optimal $K$ in the static anchor scheme is always smaller than or equal to the one in the dynamic anchor scheme, due to resetting the forwarding chain of an MC upon new session arrival in the dynamic anchor scheme.

\subsection{Proposed Schemes versus Baseline Schemes}

Fig. 9 shows the difference of the total communication cost between the proposed schemes and baseline schemes, as a

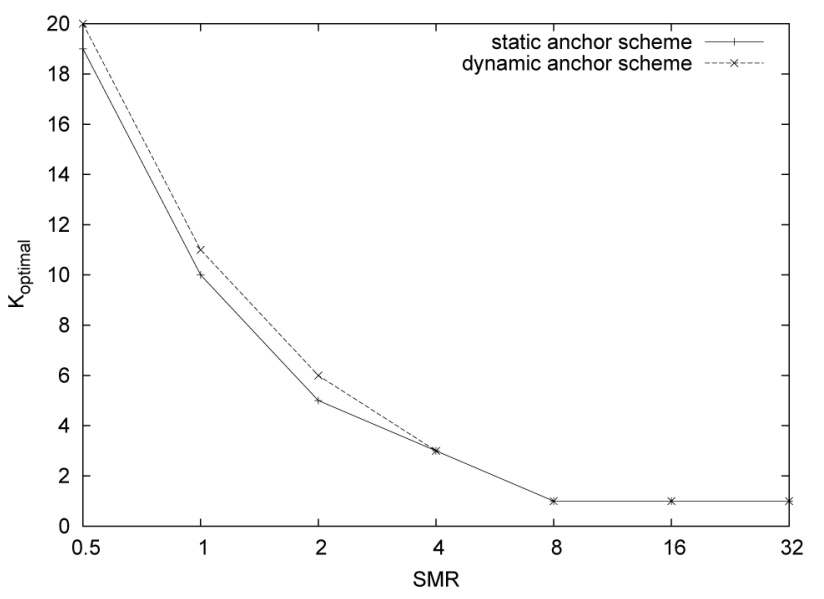

Fig. 8. Optimal $K$ versus SMR.

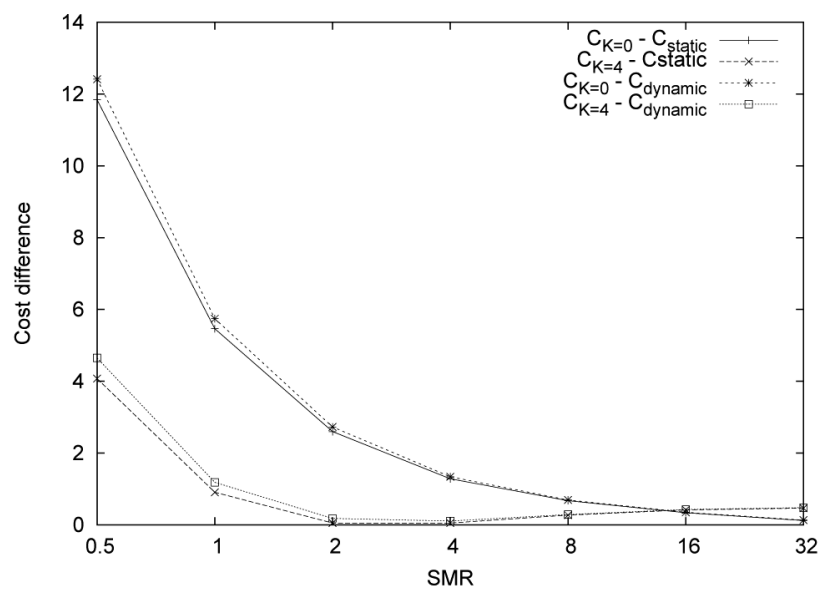

Fig. 9. Cost difference versus SMR between the proposed schemes and baseline schemes.

function of SMR. For example, $C_{K=4}-C_{\text {dynamic }}$ in the figure means the difference of the total communication cost between the dynamic anchor scheme and the baseline scheme in which a fixed threshold $K=4$ is applied to all MCs. The minimum total communication cost under the optimal threshold $K$ is used for the proposed schemes. It can be seen in the figure that the proposed schemes perform significantly better than both baseline schemes, especially when SMR is small. The cost differences decrease as SMR increases. The reason is that given fixed session arrival rates, the mobility rate and accordingly the signaling cost incurred by location management decrease as SMR increases, in both the proposed schemes and baseline schemes. As can be seen in the figure, however, the proposed schemes are always superior to both baseline schemes. The comparison demonstrates the advantage of identifying the optimal threshold of the forwarding chain length on a per-user basis.

\subsection{The WMM Scheme}

WMM [5] is a routing-based mobility management scheme, in the sense that location management is integrated with packet routing. This idea is earlier adopted by Cellular IP [17] and HAWAII [18], both of which are routing-based micromobility management schemes for Mobile IP networks. Each MR in WMM employs a proxy table to store the location information of MCs for which it has routed packets. Location information is also carried out in the IP header of every data packet. Location information is synchronized in every MR along the route of a packet between the MR's proxy table and the IP header of the packet, based on the relative magnitude of time stamps they carry.

In order to route Internet packets to an $\mathrm{MC}$, the gateway must know the location information of the MC, i.e., the address of the MC's current serving MR. If the MC's location information is not found in the gateway's proxy table, a location query procedure based on broadcasting must be executed, which incurs significant overhead. If the $M C^{\prime}$ 's location information stored in the gateway's proxy table is fresh, packets can be routed to the destination directly. On the other hand, if the stored location information is obsolete, packets will be routed to the obsolete 
serving MR first, which then forwards them to the $\mathrm{MC}^{\prime} \mathrm{s}$ current serving MR. This is similar to delivering packets by following a forwarding chain.

To route an Intranet packet, the current serving MR (MR1) of the source MC (MC1) uses different routing strategies, depending on whether its local proxy table has an entry for the destination MC (MC2). If its proxy table has an entry for MC2, MR1 routes the packet to the recorded serving MR of MC2; otherwise, it routes the packet to the gateway. In the first case, if the recorded serving MR of MC2 is obsolete, the packet will be routed to the obsolete serving MR first, which then forwards the packet to MC2's current serving MR. This is again similar to the forwarding chain approach.

Based on the above discussion, we argue that the WMM scheme can be viewed as a variant of mobility management schemes based on pointer forwarding. The first Internet packet originated from an MC after its most recent location handoff essentially serves as a location update message in the WMM scheme. Additionally, an Intranet packet originated from an MC that is routed to the gateway due to unknown location information of the destination is essentially a location update message as well. Between two consecutive (Internet or Intranet) packets originated from an $\mathrm{MC}$ arriving at the gateway, movement of the MC may trigger a series of location handoffs and a chain of proxy table entries is set up along the path of its movement. Such a chain of proxy table entries is similar to a chain of forwarding pointers. In this sense, each data packet originated from an MC arriving at the gateway essentially resets the forwarding chain, because the gateway's proxy table is updated according to the location information of the MC carried in the packet.

The average time interval between two reset operations is the same as the average interarrival time between two data packets originated from an MC reaching the gateway. Let $P_{g}$ denote the probability that an Intranet packet originated from an MC arrives at the gateway due to unknown location information of the destination. The effective arrival rate of packets originated from an MC reaching the gateway is, therefore, $\lambda_{p u}+P_{g} \times \lambda_{p L}$, and the average interarrival time $T_{l u}$ of such two consecutive packets can be calculated as

$$
T_{l u}=\frac{1}{\lambda_{p u}+P_{g} \times \lambda_{p L}} .
$$

The average distance (number of hops) an MC can move during the time interval $T_{l u}$ is, therefore, $M=T_{l u} \times \sigma$, which is essentially the threshold of the distance an MC can move between two consecutive forwarding chain reset operations. It is important to realize that, generally, the distance of movement is different from the forwarding chain length, because backward movement reduces the forwarding chain length by 1 .

Based on the above observation, we develop an SPN model for the WMM scheme, as shown in Fig. 10. Notice that we use separate places $N M$ and $F L$ to represent the distance of movement and the forwarding chain length, respectively. As discussed above, a threshold $M$ is associated with the arc from place $N M$ to transition LocUpdate. Due to the space limit, we omit the description of how the SPN model is constructed.

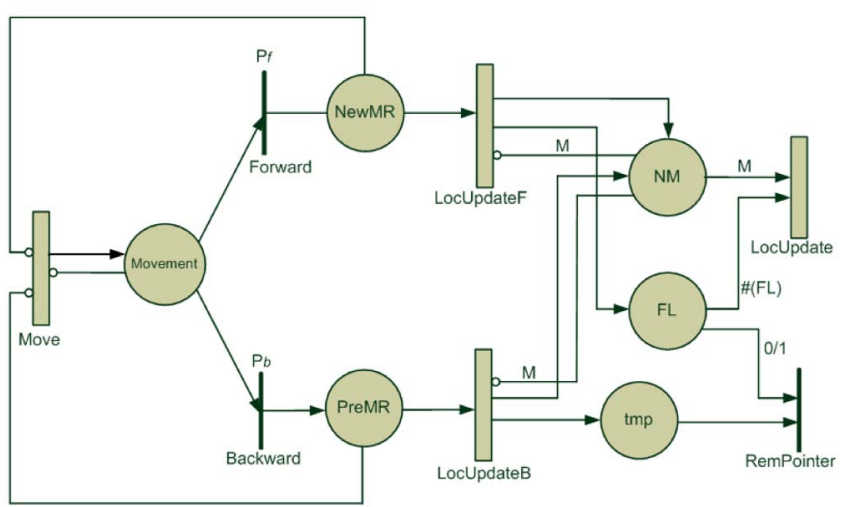

Fig. 10. The SPN model for the WMM scheme.

The total communication cost incurred by the WMM scheme, denoted by $C_{w m m}$, consists of the signaling cost incurred by handling location handoffs, the packet delivery cost, and the location query cost:

$$
\begin{aligned}
C_{\text {wmm }}= & C_{\text {location }} \times \sigma+C_{\text {delivery }, I} \times \lambda_{p d} \\
& +C_{\text {delivery }, L} \times \lambda_{p L}+C_{\text {query }} \times P_{q} \times \omega .
\end{aligned}
$$

Location handoffs are handled in the WMM scheme by a registration procedure, which involves a round-trip communication between two neighboring MRs. Therefore, $C_{\text {location }}=2 \tau$ in the WMM scheme. Let $i$ denote the number of tokens in place $F L$ in Fig. 10, i.e., $i$ is the length of the forwarding chain, then the cost of Internet packet delivery is calculated as $C_{\text {delivery, } I}=(\alpha+i) \times \tau$. Intranet sessions in WMNs, which involve two peers interacting with each other bidirectionally, usually have similar packet arrival rates in both directions. It indicates that the location information of each peer stored by the serving MR of the other peer is updated in a similar rate. Thus, packets sent and received between two peers involved in an Intranet session usually travel the same distance $\beta$ on the average. The delivery cost of Intranet packets denoted by $C_{\text {delivery }, L}$ is, therefore, $\beta \tau$ in the WMM scheme.

The location query procedure, executed by the gateway, is required only when there are packets to be sent to an MC before the MC initiates the first Internet session, after 1) the MC newly enters into a WMN or 2) the MC wakes up and reconnects to the WMN after staying in sleep mode for some time (an MC may voluntarily disconnect from a WMN and switch to sleep mode to save battery life). In both cases, packets to be sent to the MC will be routed to the gateway, because the MC's current serving MR is unknown. The probability denoted by $P_{q}$ that the location query procedure is executed in the above cases is investigated in [5]. Typically, an MC switches alternatively between active mode and sleep mode during its stay in a WMN. Let $\omega_{w}$ and $\omega_{s}$ denote the rate of switching from sleep mode to active mode and the rate of reverse mode switching, respectively. Let $\omega$ in (17) denote the reconnection rate of an MC. Then, $\omega$ can be calculated as

$$
\omega=\frac{1}{\frac{1}{\omega_{w}}+\frac{1}{\omega_{s}}}=\frac{\omega_{w} \times \omega_{s}}{\omega_{w}+\omega_{s}} .
$$




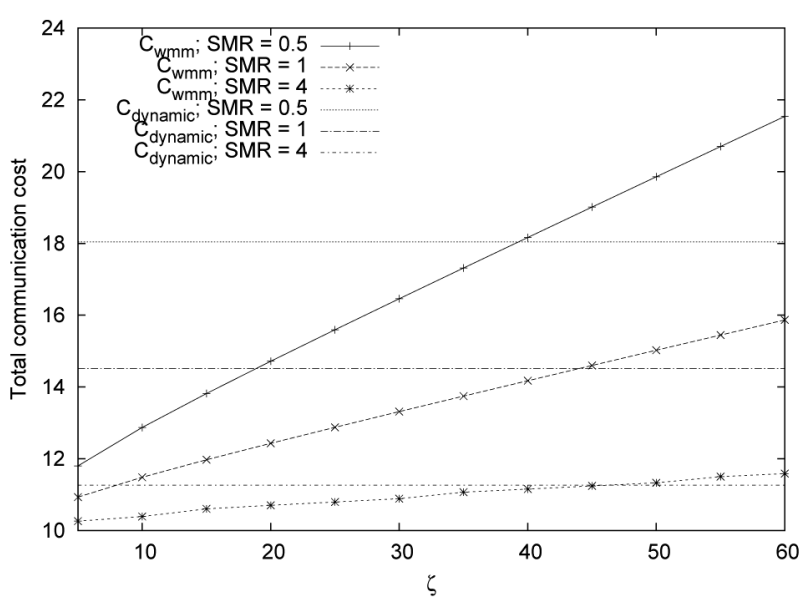

Fig. 11. Total communication cost versus $\zeta$ in the WMM scheme.

To locate an MC whose current serving MR is unknown, the location query procedure is executed by broadcasting a route request message to all MRs. The current serving MR of the $\mathrm{MC}$ replies to the gateway a route response message upon receiving the route request message. Thus, the signaling cost of the location query procedure denoted by $C_{\text {query }}$ is the sum of the cost of broadcasting the route request message and the cost of transmitting the route response message. The cost of transmitting the route response message is $\alpha \tau$. Here, we present a brief analysis of the cost of broadcasting the route request message. In the following, we define the cost of broadcasting a route request message as the number of broadcasts required to deliver the message to all MRs, instead of the sum of one-hop transmission costs, because of the broadcasting nature of wireless transmission. It is important to realize that this definition of the broadcasting cost is appropriate only by assuming that all MRs use the same omnidirectional wireless channel for communication. For future multiradio multichannel WMNs that are built upon advanced radio techniques, e.g., cognitive radio and directional antenna, the cost would be underestimated.

We assume that a flooding algorithm based on selfpruning [19] is used for broadcasting in WMNs. Selfpruning utilizes the knowledge of direct neighborhood of each node to reduce redundant rebroadcasts, which is a serious problem in flooding-based broadcasting algorithms, commonly referred to as the broadcast storm problem [20]. In self-pruning, each node $\nu$ maintains a list of direct neighbors, denoted by $N(\nu)$. A node $\nu_{j}$ who receives a flooding packet from its neighbor $\nu_{i}$ rebroadcasts the packet only if $N\left(\nu_{i}\right)-N\left(\nu_{j}\right)$ is nonempty and it is the first time $\nu_{j}$ receives the packet. This indicates that each node will rebroadcast a flooding packet no more than once. Thus, the cost of flooding the route request message in WMNs can be calculated as $P_{r} \times N$, where $P_{r}$ denotes the average probability that an MR rebroadcasts the route request message and $N$ denotes the number of MRs. Therefore, we have $C_{\text {query }}=P_{r} \times N+\alpha \tau$.

\subsection{Proposed Schemes versus the WMM Scheme}

To analyze the performance of the WMM scheme, we introduce the parameter $\zeta$, which represents the ratio of the downlink packet arrival rate to the uplink packet arrival rate of Internet sessions, based on the observation of traffic

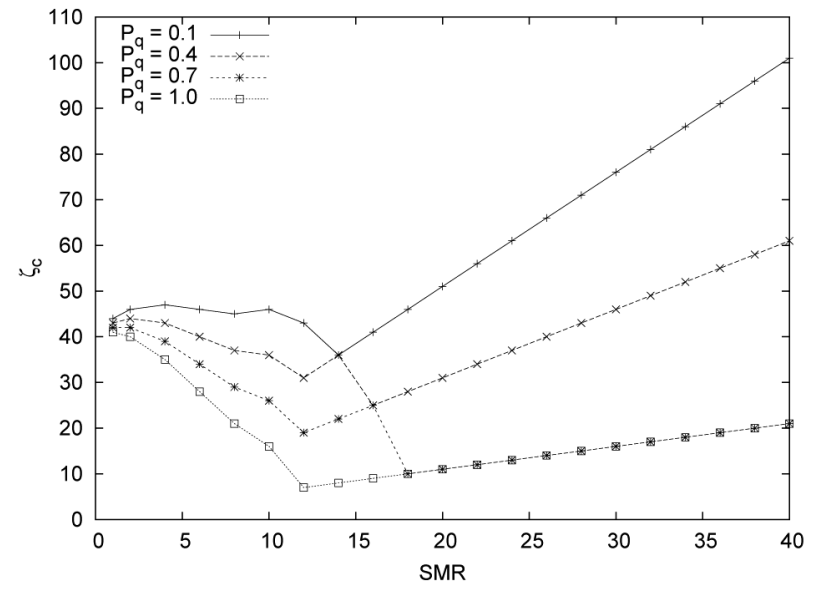

Fig. 12. $\zeta_{c}$ versus SMR.

asymmetry between the downlink and uplink [21], [22], [23], i.e., typically, the traffic load on the downlink is much larger than the one on the uplink. Traffic asymmetry is especially pronounced for mobile multimedia applications, e.g., real-time audio/video streaming, online radio, online interactive games, etc., because in such applications, small content requests are transmitted via the uplink, whereas the requested content that is typically large is transmitted via the downlink. Due to traffic asymmetry, it is expected that the downlink packet arrival rate is much higher than the uplink packet arrival rate in mobile Internet applications, i.e., $\zeta$ is expected to be reasonably large.

Fig. 11 plots the total communication cost per time unit incurred by the WMM scheme denoted by $C_{w m m}$ as a function of $\zeta$, under different SMRs. As can be seen in the figure, $C_{w m m}$ increases almost linearly as $\zeta$ increases. This is because as $\zeta$ increases, $M$ and accordingly the forwarding chain length increase, thus causing the packet delivery cost to increase. As in our schemes, $C_{w m m}$ also increases as SMR increases. It is also interesting to see in the figure that the slope of the cost curve is inversely proportional to SMR, i.e., it is in direct proportion to the mobility rate. Therefore, when the mobility rate is high, the performance of the WMM scheme degrades quickly as $\zeta$ increases. This is a major drawback of routing-based mobility management schemes, in that the propagation of updated location information of MCs relies on packet routing, thereby incurring possibly significant delay.

Fig. 11 also shows the performance of the dynamic anchor scheme under the investigated SMRs as references. The performance data of the dynamic anchor scheme shown in Fig. 11 take into consideration of the cost incurred when an MC newly enters into a WMN and each time when the $\mathrm{MC}$ wakes up and reconnects to the WMN. Let $C_{\text {reconnect }}$ denote the cost. In the proposed schemes, a location update message is sent to the gateway when either one of these two events happens. Thus, $C_{\text {reconnect }}=\alpha \tau$ in the proposed schemes. It can be seen in Fig. 11 that under a specific SMR, there exists a crossover point of $\zeta$ beyond which the dynamic anchor scheme performs better than the WMM scheme. Let $\zeta_{c}$ denote the crossover point.

Fig. 12 plots $\zeta_{c}$ as a function of SMR, under different query probabilities. It is interesting to observe that each 


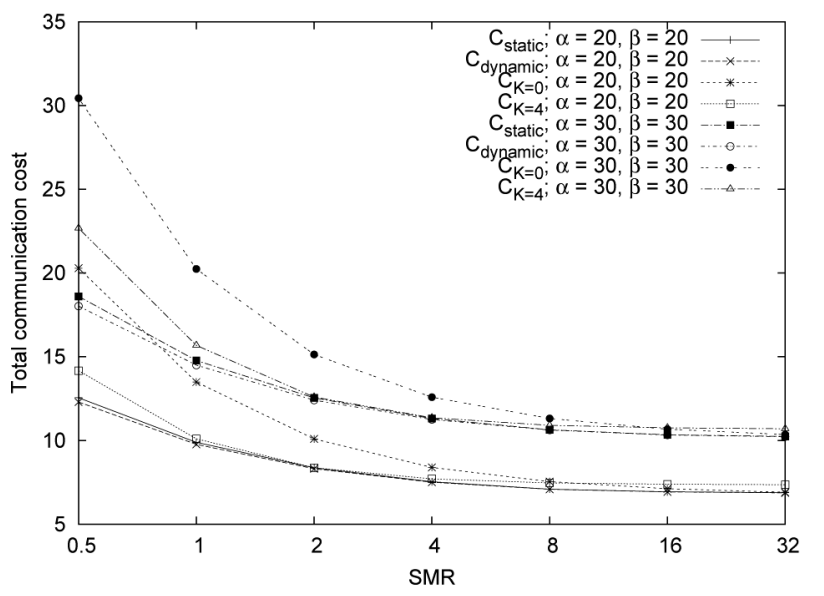

Fig. 13. Proposed schemes versus baseline schemes, under two different combinations of $\alpha$ and $\beta$.

curve in Fig. 12 exhibits a shape consisting of two segments with clearly different trends. This is the result of varying contributions to the total communication cost as SMR increases between the signaling cost incurred by location management and the service delivery cost. Specifically, when SMR is small, i.e., when the mobility rate is high, the contribution of the signaling cost incurred by location management is significant, and it is much larger in the dynamic anchor scheme than in the WMM scheme, because location update in the WMM scheme incurs minimum overhead. Therefore, when SMR is small, a relatively large $\zeta_{c}$ is necessary for the dynamic anchor scheme to yield better performance than the WMM scheme, and $\zeta_{c}$ roughly decreases as SMR increases. As SMR increases, the contribution of the signaling cost incurred by location management becomes less significant, whereas the relative contribution of the service delivery cost increases. Intuitively, there exists a point of SMR beyond which the trend is shifted, i.e., $\zeta_{c}$ starts increasing as SMR increases. As indicated above, the WMM scheme favors small mobility rates, because its performance drops quickly when the mobility rate is high. As SMR increases, the mobility rate decreases; therefore, the minimum $\zeta$ required for the dynamic anchor scheme to perform better than the WMM scheme, i.e., $\zeta_{c}$, increases.

Fig. 12 also shows that the query probability denoted by $P_{q}$ significantly affects the performance of the WMM scheme. As investigated in [5], $P_{q}$ is dependent on various parameters, and can range from lower than 5 percent to higher than 95 percent. We expect that $P_{q}$ will not be significantly low in normal network traffic conditions. For example, unless $\gamma$ is extremely large, e.g., $\gamma \geq 1,000, P_{q}$ will typically be higher than 10 percent, and may even be above 50 percent, according to the analysis of $P_{q}$ presented in [5]. Therefore, we expect that the typical range of $\zeta_{c}$ is $[10,100]$ in normal network traffic conditions. As discussed above, $\zeta$ is expected to be large for mobile Internet applications, due to traffic asymmetry between the downlink and uplink. Thus, we argue that this range is reasonable, and we conclude that the dynamic anchor scheme is superior to the WMM scheme, when mobile Internet applications dominate the network traffic in WMNs.

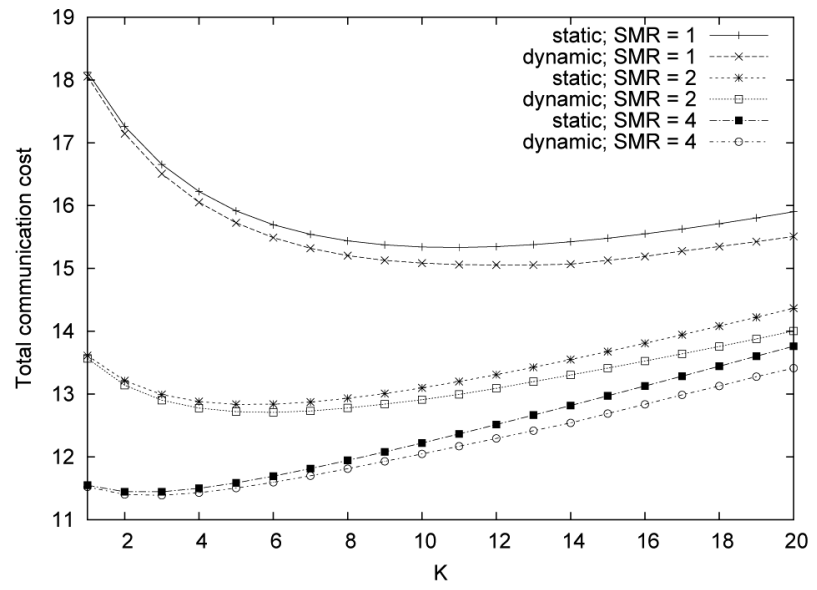

Fig. 14. Total communication cost versus $K$, assuming the hexagonal network coverage model.

\subsection{Sensitivity Analysis}

In this section, we investigate the sensitivity of analytical results with respect to various parameters characterizing the network condition and structure, e.g., $\alpha, \beta$, and the network coverage model assumed.

As can be seen in Section 6.4, $\alpha$ and $\beta$ are two critical parameters that determine the cost of mobility management as well as the cost of packet delivery. Fig. 13 compares the total communication cost between the proposed schemes and the baseline schemes, under two different combinations of $\alpha$ and $\beta$ : 1) $\alpha=20, \beta=20$ and 2) $\alpha=30, \beta=30$. As expected, under the same SMR, the total communication cost increases as $\alpha$ and $\beta$ increase. However, regardless of the values of $\alpha$ and $\beta$, the trend remains the same, i.e., the proposed schemes perform significantly better than the baseline schemes, especially when SMR is small. This observation conforms to the result illustrated in Fig. 9.

As introduced in Section 6.3, we assume that the squaregrid mesh network model for WMNs and the random walk model for MCs. In order to investigate the effect of network coverage models on the performance of the proposed schemes, we switch to the hexagonal network coverage model as used in [5]. In the hexagonal network coverage model, the coverage area of each MR is called a cell, and each MR has six direct neighbors. An MC can move randomly from an MR to one of its direct neighbors with the same probability. Thus, $P_{f}=\frac{5}{6}$ and $P_{b}=\frac{1}{6}$, under the hexagonal network coverage model.

Fig. 14 plots the total communication cost incurred by the proposed schemes as a function of $K$, under different SMRs, assuming the hexagonal network coverage model. Comparing Figs. 6 and 14, it can be seen that cost curves shown in both figures exhibit high similarity in shape. Therefore, we can draw the conclusion that analytical results obtained are valid and are not sensitive to the network coverage model.

\section{CONCLUSION}

In this paper, we propose two mobility management schemes based on pointer forwarding for wireless mesh networks, namely, the static anchor scheme and dynamic anchor scheme. The proposed schemes are per-user-based, 
in that the optimal threshold of the forwarding chain length that minimizes the total communication cost is dynamically determined for each individual MC, based on the MC's specific mobility and service patterns characterized by SMR.

We develop analytical models based on stochastic Petri nets to evaluate the performance of the proposed schemes. We also compare the proposed schemes with two baseline schemes and with the WMM scheme. Analytical results show that 1) the dynamic anchor scheme is better than the static anchor scheme in typical network traffic conditions, whereas the static anchor scheme is better when the service rate of an MC is comparatively high such that the advantage of the dynamic anchor scheme is offset by the extra cost; 2) our schemes perform significantly better than the baseline schemes, especially when SMR is small; and 3) the dynamic anchor scheme is superior to the WMM scheme when the network traffic is dominated by mobile Internet applications characterized by large traffic asymmetry for which the downlink packet arrival rate is much higher than the uplink packet arrival rate.

In the future, we plan to investigate how our proposed schemes can be extended to WMNs that have multiple gateways. In addition, we plan to investigate the proposed schemes under more realistic mobility models other than the random walk model. We will also investigate how caching of location information of MCs can be used to reduce the signaling cost incurred by our proposed schemes.

\section{REFEREnCES}

[1] I.F. Akyildiz, X. Wang, and W. Wang, "Wireless Mesh Networks: A Survey," Computer Networks, vol. 47, no. 4, pp. 445-487, Mar. 2005.

[2] A. Raniwala and T.-c. Chiueh, "Architecture and Algorithms for an ieee 802.11-Based Multi-Channel Wireless Mesh Network," Proc. IEEE INFOCOM, vol. 3, pp. 2223-2234, Mar. 2005.

[3] I. Akyildiz, J. McNair, J. Ho, H. Uzunalioglu, and W. Wang, "Mobility Management in Next-Generation Wireless Systems," Proc. IEEE, vol. 87, no. 8, pp. 1347-1384, Aug. 1999.

[4] I. Akyildiz, J. Xie, and S. Mohanty, "A Survey of Mobility Management in Next-Generation All-IP-Based Wireless Systems," IEEE Wireless Comm., vol. 11, no. 4, pp. 16-28, Aug. 2004.

[5] D. Huang, P. Lin, and C. Gan, "Design and Performance Study for a Mobility Management Mechanism (WMM) Using Location Cache for Wireless Mesh Networks," IEEE Trans. Mobile Computing, vol. 7, no. 5, pp. 546-556, May 2008.

[6] A. Boukerche and Z. Zhang, "A Hybrid-Routing Based IntraDomain Mobility Management Scheme for Wireless Mesh Networks," Proc. 11th Int'l Symp. Modeling, Analysis and Simulation of Wireless and Mobile Systems (MSWiM '08), pp. 268-275, Oct. 2008.

[7] H. Wang, Q. Huang, Y. Xia, Y. Wu, and Y. Yuan, "A NetworkBased Local Mobility Management Scheme for Wireless Mesh Networks," Proc. IEEE Wireless Comm. and Networking Conf. (WCNC '07), pp. 3792-3797, Mar. 2007.

[8] R. Huang, C. Zhang, and Y. Fang, "A Mobility Management Scheme for Wireless Mesh Networks," Proc. 50th IEEE Global Telecomm. Conf., pp. 5092-5096, Nov. 2007.

[9] V. Navda, A. Kashyap, and S. Das, "Design and Evaluation of iMesh: An Infrastructure-Mode Wireless Mesh Network," Proc. Sixth IEEE Int'l Symp. World of Wireless Mobile and Multimedia Networks (WoWMoM'05), pp. 164-170, June 2005.

[10] M. Ren, C. Liu, H. Zhao, T. Zhao, and W. Yan, "MEMO: An Applied Wireless Mesh Network with Client Support and Mobility Management," Proc. 50th IEEE Global Telecomm. Conf., pp. 5075-5079, Nov. 2007.

[11] Y. Amir, C. Danilov, M. Hilsdale, R. Musaloiu-Elefteri, and N. Rivera, "Fast Handoff for Seamless Wireless Mesh Networks," Proc. MobiSys, pp. 83-95, June 2006.
[12] R. Jain and Y. Lin, "An Auxiliary User Location Strategy Employing Forwarding Pointers to Reduce Network Impacts of PCS," Wireless Networks, vol. 1, no. 2, pp. 197-210, 1995.

[13] N. Nandiraju, L. Santhanam, B. He, J. Wang, and D. Agrawal, "Wireless Mesh Networks: Current Challenges and Future Directions of Web-in-the-Sky," IEEE Wireless Comm., vol. 14, no. 4, pp. 79-89, Aug. 2007.

[14] J. Robinson and E. Knightly, "A Performance Study of Deployment Factors in Wireless Mesh Networks," Proc. IEEE INFOCOM, pp. 2054-2062, May 2007.

[15] I.F. Akyildiz, Y.-B. Lin, W.-R. Lai, and R.-J. Chen, "A New Random Walk Model for PCS Networks," IEEE J. Selected Areas in Comm., vol. 18, no. 7, pp. 1254-1260, July 2000.

[16] C. Hirel, B. Tuffin, and K.S. Trivedi, "SPNP: Stochastic Petri Nets, Version 6.0," Proc. 11th Int'l Conf. Computer Performance Evaluation: Modelling Techniques and Tools, pp. 354-357, Mar. 2000.

[17] A. Campbell, J. Gomez, S. Kim, A. Valko, C. Wan, and Z. Turanyi, "Design, Implementation, and Evaluation of Cellular IP," IEEE Personal Comm., vol. 7, no. 4, pp. 42-49, Aug. 2000.

[18] R. Ramjee, T.L. Porta, S. Thuel, K. Varadhan, and S. Wang, "HAWAII: A Domain-Based Approach for Supporting Mobility in Wide-Area Wireless Networks," Proc. Seventh Int'l Conf. Network protocols (ICNP '99), pp. 283-292, 1999.

[19] H. Lim and C. Kim, "Flooding in Wireless Ad Hoc Networks," Computer Comm., vol. 24, nos. 3/4, pp. 353-363, 2001.

[20] S.-Y. Ni, Y.-C. Tseng, Y.-S. Chen, and J.-P. Sheu, "The Broadcast Storm Problem in a Mobile Ad Hoc Network," Proc. Ann. ACM/ IEEE MobiCom, pp. 151-162, Aug. 1999.

[21] D.G. Jeong and W.S. Jeon, "CDMA/TDD System for Wireless Multimedia Services with Traffic Unbalance between Uplink and Downlink," IEEE J. Selected Areas in Comm., vol. 17, no. 5, pp. 939946, May 1999.

[22] W.S. Jeon and D.G. Jeong, "Call Admission Control for Mobile Multimedia Communications with Traffic Asymmetry between Uplink and Downlink," IEEE Trans. Vehicular Technology, vol. 50, no. 1, pp. 59-66, Jan. 2001.

[23] I. Sohn, B.O. Lee, and K.B. Lee, "Balancing Uplink and Downlink under Asymmetric Traffic Environments Using Distributed Receive Antennas," IEICE Trans. Comm., vol. E91-B, no. 10, pp. 3141-3148, 2008.

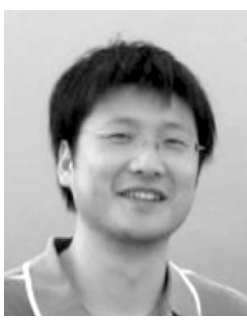

Yinan Li received the BS degree in computer science from Xi'an Jiaotong University, China, and the MS degree in computer science from the University of Tennessee in 2008 . He is currently working toward the $\mathrm{PhD}$ degree from the Department of Computer Science at the Virginia Polytechnic Institute and State University. His research interests include wireless networks, mobile ad hoc networks, sensor networks, network security, high-performance computing, and dependable computing.

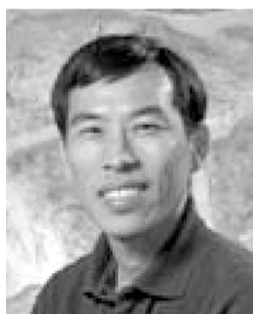

Ing-Ray Chen received the BS degree from the National Taiwan University, Taipei, and the MS and $\mathrm{PhD}$ degrees in computer science from the University of Houston. He is a professor in the Department of Computer Science at the Virginia Polytechnic Institute and State University. His research interests include mobile computing, wireless networks, security, multimedia, realtime intelligent systems, and reliability and performance analysis. He is currently serving as an editor for The Computer Journal, Wireless Personal Communications, Wireless Communications and Mobile Computing, Security and Communication Networks, and the International Journal on Artificial Intelligence Tools. He is a member of the IEEE, the IEEE Computer Society, and the ACM.

$\triangleright$ For more information on this or any other computing topic, please visit our Digital Library at www.computer.org/publications/dlib. 